\title{
Overcoming pain thresholds with multilevel models - An example using Quantitative Sensory Testing (QST) data
}

Gerrit Hirschfeld, Markus R Blankenburg, Moritz Süß, Boris Zernikow

The assessment of somatosensory function is a cornerstone of research and clinical practice in neurology. Recent initiatives have developed novel protocols for quantitative sensory testing (QST). Application of these methods led to yielded intriguing findings, such as the presence lower pain-thresholds in healthy children compared to healthy adolescents. In this article, we (re-) introduce the basic concepts of signal detection theory (SDT) as a method to investigate such differences in somatosensory function in detail. SDT describes participants' responses according to two parameters, sensitivity and responsebias. Sensitivity refers to individuals' ability to discriminate between painful and nonpainful stimulations. Response-bias refers to individuals' criterion for giving a "painful" response. We describe how multilevel models can be used to estimate these parameters and to overcome central critiques of these methods. To provide an example we apply these methods to data from the mechanical pain sensitivity test of the QST protocol. The results show that adolescents are more sensitive to mechanical pain and contradict the idea that younger children simply use more lenient criteria to report pain. Overall, we hope that the wider use of multilevel modeling to describe somatosensory functioning may advance neurology research and practice. 


\section{Overcoming pain thresholds with multilevel models -} An example using Quantitative Sensory Testing (QST) data

Address for correspondence: Prof. Dr. Gerrit Hirschfeld; Faculty of Business Management and

21 Social Sciences, University of Applied Sciences Osnabrück, Osnabrueck, Caprivistr. 30 A,

2249076 Osnabrück, Germany; eMail: hirschfeld@wi.hs-osnabrueck.de 


\section{Abstract}

25 The assessment of somatosensory function is a cornerstone of research and clinical practice in

26 neurology. Recent initiatives have developed novel protocols for quantitative sensory testing

27 (QST). Application of these methods led to yielded intriguing findings, such as the presence

28 lower pain-thresholds in healthy children compared to healthy adolescents. In this article, we (re-

29 ) introduce the basic concepts of signal detection theory (SDT) as a method to investigate such

30 differences in somatosensory function in detail. SDT describes participants' responses according

31 to two parameters, sensitivity and response bias. Sensitivity refers to individuals' ability to

32 discriminate between painful and non-painful stimulations. Response bias refers to individuals'

33 criterion for giving a "painful" response. We describe how multilevel models can be used to

34 estimate these parameters and to overcome central critiques of these methods. To provide an

35 example we apply these methods to data from the mechanical pain sensitivity test of the QST

36 protocol. The results show that adolescents are more sensitive to mechanical pain and contradict

37 the idea that younger children simply use more lenient criteria to report pain. Overall, we hope

38 that the wider use of multilevel modeling to describe somatosensory functioning may advance

39 neurology research and practice.

42 Keywords: pain; thresholds; signal detection theory; sensitivity; multilevel models;

\section{3 quantitative sensory testing}




\section{Introduction}

46 The assessment of somatosensory function is a cornerstone of research and clinical practice in

47 neurology. Understanding both acute as well as chronic pain relies, to a large extent, on our

48 ability to measure and quantify the response to painful and non-painful stimuli. Many resources

49 have been used to develop novel and standardized methods to collect such data, e.g. the

50 quantitative sensory testing (QST) protocol of the German research network on neuropathic pain

51 (DFNS)(Rolke et al., 2006). The QST protocol has been adapted for other languages

52 (Schestatsky et al., 2011) and children (Blankenburg et al., 2010). The availability of such

53 standardized measures has resulted in a rapid increase in the number of studies into pain

54 processing in healthy participants and patients. These experimental paradigms have also been

55 used to investigate long-standing observations in clinical practice, e.g. higher pain-ratings in

56 women compared to men (Fillingim et al., 2009) or higher pain-ratings in girls compared to boys

57 (Goodenough et al., 1999). While some of these clinical findings can be replicated in

58 experimental paradigms, researchers have also identified some differences. In pediatric settings,

59 girls report higher pain-intensity ratings following venipuncture than boys (Goodenough et al.,

60 1999), while such differences are not apparent in QST data (Blankenburg et al., 2011). This may

61 be because experimental studies describe participants' responses exclusively in terms of

62 thresholds that conflate several aspects of the response. One method to tease out several aspects

63 of participants' responses is signal detection theory (SDT). While SDT has been criticized in the

64 past (Clark, 1974; Rollman, 1976; Chapman, 1977; Gracely, 2006), we believe that several

65 recent developments concerning the standardization of QST protocols and data-analysis should

66 lead to a reassessment of SDT. 
67 The aim of the present paper is to re-introduce the basic concepts of (SDT) and show how

68 modern multilevel models can be used to estimate the two SDT parameters, sensitivity and

69 response bias, from the mechanical pain sensitivity test within the QST protocol of the DFNS.

70 1.1. Thresholds

71 The most intuitive and widely used way to describe participants' pain reports is in terms of

72 thresholds (Gracely, 2006). Pain thresholds are typically defined as the stimulus intensity at

73 which participants report experiencing pain in 50 percent of the trials or the intensity at which

74 animals show a withdrawal response in 50 percent of the trials (Mills et al., 2012). There are two

75 different methods to determine pain thresholds; the methods of constant stimuli (also known as

76 the method of levels) and the method of limits. When the method of constant stimuli is used,

77 participants are presented with stimuli that are above and below the pain-threshold. After each

78 stimulus participants report whether the stimulus was painful or not. The threshold is determined

79 as the stimulus intensity at which $50 \%$ of the stimuli are rated as painful. The main disadvantage

80 of this method is that it takes some time to present all different stimulus intensities. This may

81 result in fatigue and sensitization that both affect the measurement. That is why most stimulation

82 protocols for clinical application use the more time-efficient method of limits, i.e. alternating

83 between a gradually increasing or decreasing series of stimulus intensities until a participant

84 changes the response from non-painful to painful, or vice versa. The threshold is determined as

85 the stimulus intensity at which participants change their response. The problems with the method

86 of limits becomes apparent when it is applied to other domains of sensory testing, e.g. visual

87 acuity testing (figure 1). Because it is very easy for participants to predict upcoming stimuli, they

88 can easily fake their test results. Furthermore, when the intensity of stimuli can only by gradually

89 increased, e.g. heat when determining heat-pain thresholds, participants' reaction time will affect 
90 the thresholds. Specifically, when the heat is increased until participants report a painful

91 response, participants with fast reaction times will appear to have lower heat-pain thresholds than

92 participants with slow reaction times.

93
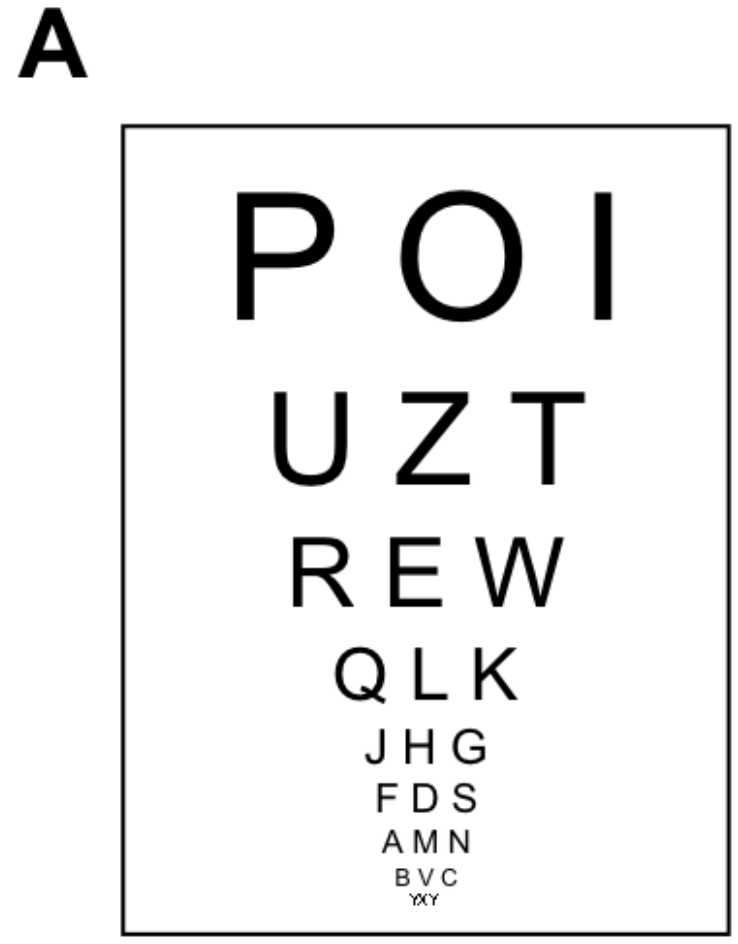

Read aloud from top to bottom.
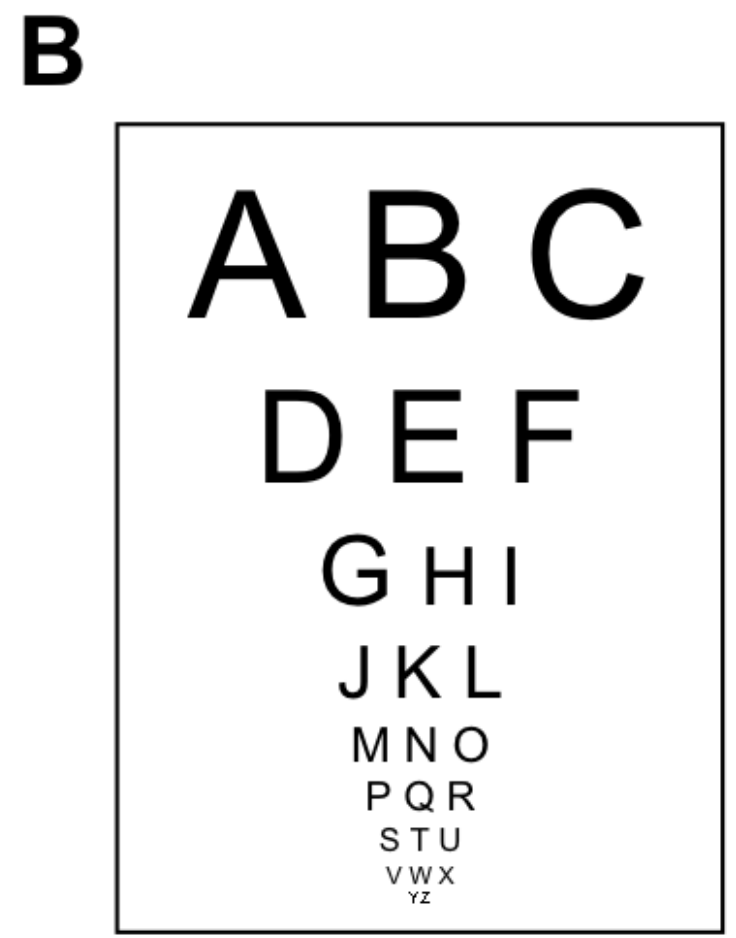

\section{Read aloud from top to bottom.}

95 Figure 1. Problems associated with the method of limits. (A) Analogue to the method of

96 constant stimuli: Randomized order of stimuli, as in the method of constant stimuli, prohibits any

97 predictions. (B) Analogue to the method of limits: Ordered presentation makes predicting the

98 next stimulus extremely easy. 
100

101

102

103

104

105

106

107

108

109

110

111

112

113

114

115

116

117

118

119

120

121

122

\subsection{Signal detection theory}

An alternative to describing participants' responses in terms of thresholds is to use signal detection theory to model their responses within individual trials (Green \& Swets, 1966;

Macmillan \& Creelman, 2004). However, to use this method, data need to be collected using the method of constant stimuli (gathering individual ratings on a random series of stimuli, including empty trials without stimulation). According to signal detection theory, participants' responses can be modeled by two parameters, sensitivity and response bias. Sensitivity refers to the ability to accurately discriminate between the presence and absence of a target stimulus. Response bias refers to participants' criteria for reporting the presence or absence of the stimulus. Differences in these parameters become apparent when stimulus-response-functions are plotted for a number of participants (see fig. 2 below). Participants with high sensitivity show a steep increase in the percent of painful responses with increasing stimulus intensities. Participants with high criteria for pain show these increases at higher stimulus intensities. The most widely used metrics for sensitivity and response bias are d' and C, respectively. These two parameters are readily calculated from a $2 \mathrm{X} 2$ table consisting of the counts for hits (participants correctly report presence of stimuli), misses (participants report absence of stimuli even though they are present), false-alarms (participants report presence of stimuli even though they are absent), and correct rejections (participants correctly report absence of stimuli) (Green \& Swets, 1966). From these the hit rate $[$ hits/(hits + misses) $]$ and false-alarm rate $[$ false-alarms / (false alarms + correct rejections)] are computed. The measure d' is defined as the z-score corresponding to the hit rate minus the z-score corresponding to the false-alarm rate. If participants are very good at distinguishing the presence or absence of stimuli, they have many hits and correct rejections and few false-alarms and misses resulting in high values for d'. If participants are bad at 
123 distinguishing the presence or absence of stimuli and perform at chance level, d' will be zero.

124 The measure $\mathrm{C}$ is defined as -.5(-score corresponding to the hit rate plus the $\mathrm{z}$-score

125 corresponding to the false-alarm rate). If participants show no preference for either response, $\mathrm{c}$ is

126 zero. If participants have a preference towards a specific response this will be either positive or

127 negative. More information on the calculation and interpretation of SDT measures is given by

128 Stansislaw and Todorov (1999).

129 Importantly, it is much more difficult for participants to willingly change the test-results

130 into a specific direction. First, because the SDT measures d' and C are much less transparent to

131 participants than thresholds determined using the method of limits. With the latter most

132 participants will understand that they only have to report "painful" to light touch to fake sings of

133 a painful condition. However, only very few will have a profound-enough understanding of SDT

134 to change the sensitivity to pain as determined by SDT towards a specific direction. Second,

135 because in analogy to visual acuity testing, it is impossible to correctly identify the letters if they

136 are not discernable. That is it is impossible to fake being able to discern specific stimuli isf in

137 reality one cannot do so.

138 Crawford Clark was among the first to apply the SDT model to pain assessment (Clark,

139 1974). This initiated a highly active and controversial discussion about the application of

140 psychophysical methods to pain research in the 1970s and 1980s (Clark, 1974; Rollman, 1976;

141 Chapman, 1977; Gracely, 2006). Clark’s contention that sensitivity is only affected by

142 "neurologic" factors, while response bias is only affected by "psychologic" factors, made this

143 purely descriptive approach at the same time highly interesting and controversial. Using this

144 dichotomy, he demonstrated that interventions, such as acupuncture, only affect response bias

145 but not sensitivity (Clark \& Yang, 1974). Similarly, he found that chronic pain patients differ 
146 from healthy controls in that they use more stoical criteria for painful stimuli (Yang et al., 1985).

147 There were, however, many criticisms for this approach and especially Clark's assumption that

148 the two SDT-parameters are influenced only by the supposed factors (Rollman, 1979).

149 Ultimately, this assumption did not stand up to further empirical tests. For example, Gracely and 150 colleagues showed that "psychologic" factors may affect sensitivity (Gracely, 2006). However,

151 SDT is still a useful framework for describing participants' performance, even though Clark's

152 strict interpretation of these parameters as indexes for "psychologic" and "neurologic" factors is 153 likely wrong.

154 Additionally, two technical problems averted the wider use of these techniques. First,

155 there were no established protocols that would specify the mode of stimulation and intensity. As

156 has been noted before using different methods results in incomparable findings across studies

157 (Rollman, 1976). However, the introduction of the DFNS-QST protocol has homogenized the

158 methods to collect data that can be analyzed using SDT. Specifically the mechanical pain

159 sensitivity test lends itself easily for an analysis in terms of SDT (Rolke et al., 2006). Second, if

160 the SDT parameters are estimated via 2 X2 tables as introduced by Clark and colleagues, only

161 binary responses and two levels of stimuli can be processed. That is, even if several stimulus

162 intensities are used, these have to be dichotomized into painful and non-painful stimuli, resulting

163 in a loss of variance and possibly different effects, depending on the chosen cutpoint (Rollman,

164 1976). However, several recent extensions of multilevel models (Wright, Horry \& Skagerberg,

165 2009; Wright \& London, 2009) make it possible to estimate the SDT-parameters using the full 166 information conveyed in participants' responses. 
167

168

169

170

171

172

173

174

175

176

177

178

179

180

181

182

183

184

185

186

187

188

\subsection{Multilevel models}

It has been known for some time that generalized linear models (GLMs) can be used to estimate the SDT-parameters (DeCarlo, 1998). Namely, the slope and intercept of a probitregression function are analogues to the d' and $C$ parameters estimated by traditional SDT. As long as the stimulus and response variables are dichotomized, these will provide the numerically same results as the approach based in $2 \mathrm{X} 2$ tables (Wright \& London, 2009). The biggest advantage is, however, that multilevel models can also address continuous stimuli and responses. Specifically, using continuous measures for stimulation circumvents the above-mentioned problem that there are no objective criteria to judge whether pain is present or absent. Furthermore, if these models are fit in a multilevel context, these techniques are much more flexible in terms of the collected data and group-differences that can be tested.

While such methods were only previously available in highly specialized software, there are a number of implementations to fit GLMs using the open-source software R (R Core Team, 2012). Currently, the most widely used implementation to fit GLMs is the lme4 package (Bates \& Sarkar, 2007) and specialized packages that access functions from the LME4 package to fit psychophysical models (Wright, Horry \& Skagerberg, 2009).

\section{Example application: Age and gender effects in mechanical pain sensitivity}

\subsection{Methods}

In order to examine the effects of age and gender on mechanical pain sensitivity we reanalyzed data from 172 healthy children $(n=85 ; 41$ female) and adolescents $(n=87 ; 41$ female) who took part in an earlier study (fig. 2) (Blankenburg et al., 2011). The study was approved by 
189 the Ethics Committee of the Witten/ Herdecke University (101/2008) and encompassed written

190 informed consent from children and their guardians.

191 Participants completed the QST protocol of the German Research Network on

192 Neuropathic Pain (Rolke et al., 2006) adopted for children (Blankenburg et al., 2010). The QST

193 protocol consists of thirteen tests that assess both nociceptive as well as non-nociceptive

194 modalities and afferent nerve fibers and central pathways. The whole protocol is widely used to 195 study both healthy subjects and patients (Magerl et al., 2010; Maier et al., 2010; Mücke et al., 196 2014) as well as children (Blankenburg et al., 2011; Hirschfeld et al., 2012). Here we analyzed 197 the data from the Mechanical Pain Sensitivity (MPS) test. Within the MPS six different pinprick 198 mechanical stimulators with weights between $8 \mathrm{mN}$ and $256 \mathrm{mN}$, and three light tactile

199 stimulators (cotton wisp, cotton wool tip and brush) were applied to the back of both hands in 200 pseudorandomized sequence. Each of the nine stimulators was applied ten times (five times on 201 each hand) resulting in 90 ratings for each child and a total of 15.480 ratings for all children. The analysis proceeded in two steps: model building and assessment. First, we specified a baseline model that predicted the response (painful or not painful) using only a constant as a 204 fixed effect and participants and stimulus intensity as random effects (Barr et al., 2013). The random effect for participant indexes the individual intercept that can be interpreted in terms of response bias with high values indicating that participants were more likely to report a painful response. The random effect for stimulus intensity indexes the individual slope parameter and may be interpreted in terms of the sensitivity. Specifically, high values on the slope parameter indicate that participants show a steep increase in painful responses with increasing stimulus

210 intensity. Conversely, low values indicate that participants show only a weak increase in painful 211 responses with increasing stimulus intensity. We then sequentially added stimulus intensity, age 
212 and sex as fixed effects. These fixed effects describe effects that hold for the whole group of

213 participants, i.e. the fixed effect for stimulus intensity describes that across all participants high

214 stimulus intensities were more likely to elicit a "painful" response. For each effect that was

215 added we checked whether the inclusion significantly improved the model fit (Baayen, Davidson

216 \& Bates, 2008). Only effects that resulted in a significant improve the model fit were retained in

217 the final model. Second, we inspected the final model and extracted information on the fixed

218 effects, because these describe relevant effects at the group level. These steps are readily

219 implemented in R (see table1 for an overview of relevant functions).

220

221 Table 1. Important functions to fit GLMs to psychophysical data.

\begin{tabular}{ll}
\hline \multicolumn{1}{c}{ Function } & \multicolumn{1}{c}{ What it does } \\
\hline glmer() & Fits a model to data. The example code specifies a dependent \\
& variable "res_01", fixed effects "Stimulus", random effects \\
& "(Stimulus|Code)", a link function "binomial(link=logit)", and the \\
& data to which this is fitted. \\
& Example: \\
& $>$ mod_01<-glmer(res_01 Stimulus + (Stimulus|Code), family = \\
& binomial(link=logit), data = data) \\
& Compares fitted models to each other. The example code compares \\
& three consecutively more complex models. \\
& Example: \\
& $>$ anova(mod_01, mod_02, mod_03) \\
& Gives an overview of the model, including parameter estimates and \\
& significance levels. \\
& Example: \\
& $>$ summary(mod_03) \\
summary() & Prints the random and fixed effects. \\
ranef() and fixef() &
\end{tabular}




\section{Example: \\ $>$ ranef(mod_3) \\ $>$ fixef(mod3)}

222

\section{2.2. Results}

224 In a first step the responses by individual participants were visualized to inspect whether

225 the responses conform to the assumed model or not. As can be seen in figures 2 and 3 the model

226 fitted the responses of individual participants pretty well.

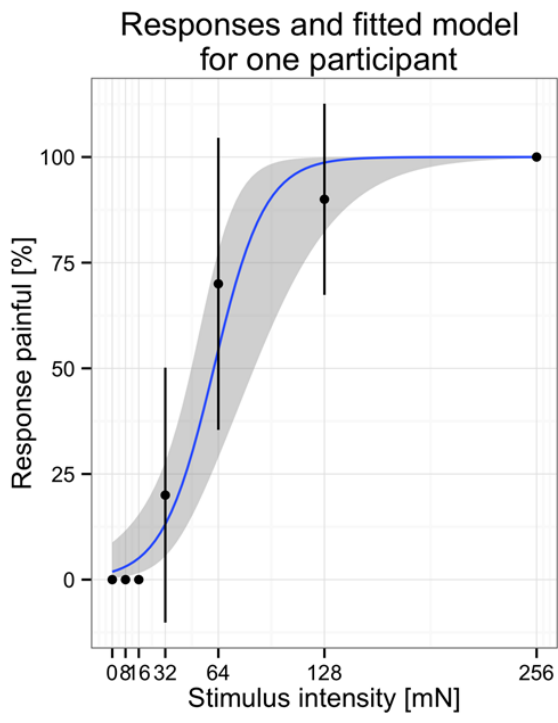

228 figure 2. Responses and fitted model for one subject. Participants rated stimuli at different

229 intensities. Note: Points represent the average \% of responses painful at each stimulus intensity, 230 the blue line indicates the fit to the individual data 
Responses and fitted model for all participants
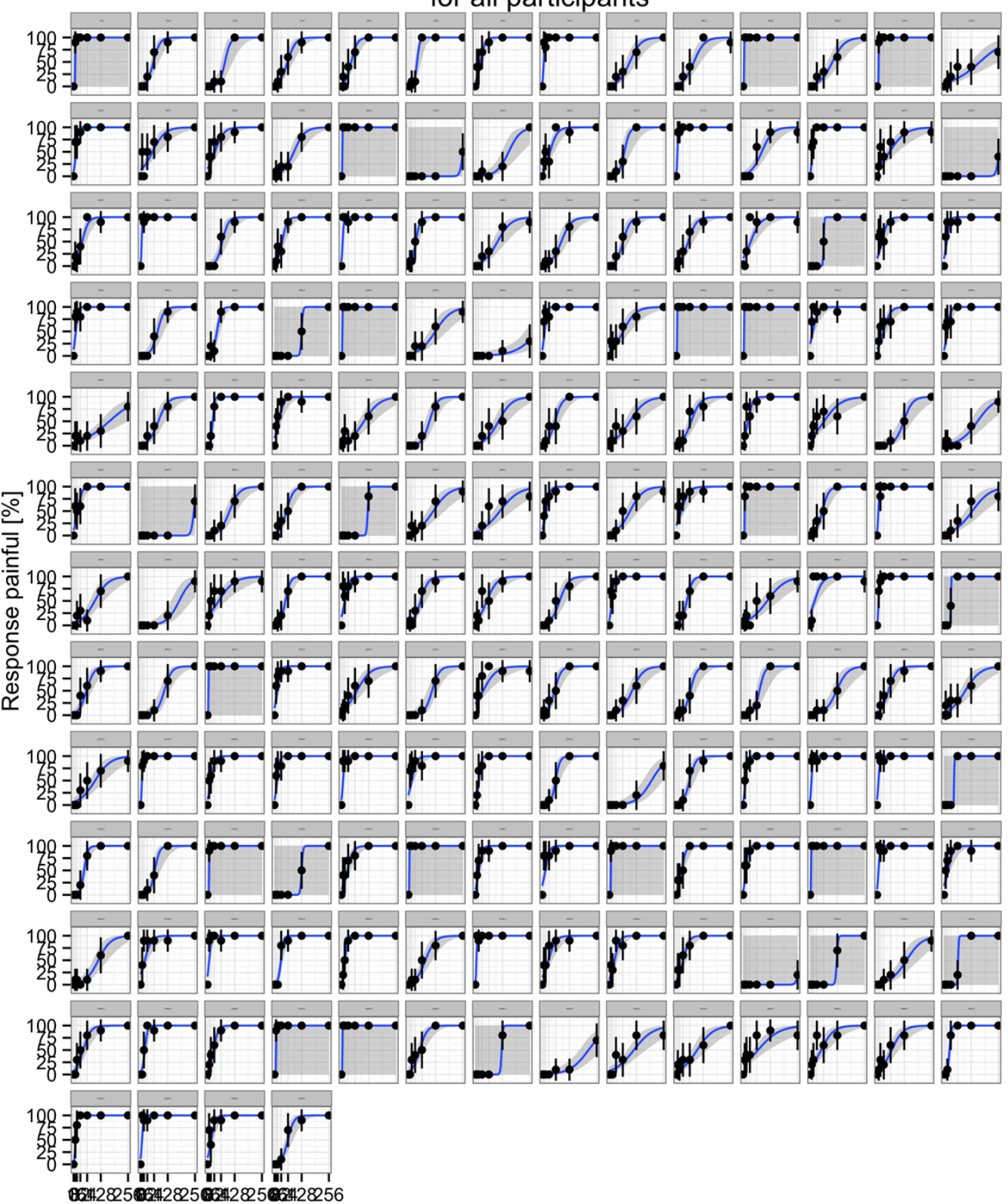

Stimulus intensity $[\mathrm{mN}]$

232 Figure 3. Responses of individual participants. Each panel represents an individual, points

233 represent the average $\%$ of responses painful at each stimulus intensity, the blue line indicates the

234 fit to the individual data. 
237 (tab. 2). Adding stimulus intensity as a fixed effect to the baseline model greatly improved the

238 model fit $\left(\chi^{2}=94.7 ; \mathrm{df}=1 ; \mathrm{p}<.001\right)$. Adding sex and the interaction between sex and stimulus

239 intensity as a fixed effect to this model did not result in a significant increase in model fit $\left(\chi^{2}=\right.$

$2401.79 ; \mathrm{df}=2 ; \mathrm{p}<.41)$; therefore, these effects were not added to the model. This indicates that

241 there were no systematic differences in sensitivity and response bias between boys and girls.

242 Adding age and the interaction between age and stimulus intensity to the model significantly

243 improved the model fit $\left(\chi^{2}=8.85 ; \mathrm{df}=2 ; \mathrm{p}=.01\right)$, indicating age-differences in either the slope

244 or intercept of the function.

246 Table 2. Series of model comparisons

\begin{tabular}{llll}
\hline Model & $\chi^{2}\left(\operatorname{diff} \chi^{2}\right)$ & DF (diff DF) & $\mathrm{p}\left(\chi^{2}\right)$ \\
\hline M1: Baseline & & & \\
M2: Model M1 plus stimulus & $(94.7011)$ & 1 & $<.001$ \\
M3: Model M2 plus sex and stimulus * sex & $(1.7872)$ & 2 & 0.41 \\
M4: Model M2 plus age and stimulus * age & $(8.847)$ & 2 & .01 \\
\hline
\end{tabular}

247

Inspection of the resulting model parameters (tab. 3) revealed that only the main-effect

249 for stimulus $(\mathrm{OR}=1.11 ; \mathrm{p}<.001)$ and the interaction between age and stimulus intensity was

250 significant $(\mathrm{OR}=1.07 ; \mathrm{p}=.003)$. The main effect for stimulus intensity showed that participants

251 were more likely to report that pinpricks were painful when these had high $\mathrm{mN}$. The larger the

252 effect for stimulus intensity the steeper the slope of the response curve. This may be interpreted

253 as better discrimination between different stimulus intensities. Similarly the interaction between 
254 age and stimulus intensity shows that the slope of the response curve is steeper in adolescents 255 compared to children. This may be interpreted as a better discrimination in adolescents. Absence 256 of the main-effect for age indicates that no differences in response bias were present.

257

258 Table 3. Estimates of the final model (M4)

\begin{tabular}{lccccc}
\hline Parameter & Estimate & SE & OR & Z & $p$ \\
\hline Intercept & -3.29 & .14 & 0.04 & -23.11 & $<.001$ \\
Stimulus & 0.10 & .02 & 1.11 & 6.20 & $<.001$ \\
Age (Children) & 0.14 & .20 & 1.15 & .72 & .473 \\
Stimulus * Age & .07 & .02 & 1.07 & 2.97 & .003 \\
\hline
\end{tabular}

Note: Reference category in parenthesis

\section{Discussion}

262 The development and use of the QST protocol of the DFNS has revived interest in the methods

263 used to collect these responses (Rolke et al., 2006). At the same time, methods to analyze

264 participants' responses in such paradigms have not followed suit. Most studies in pain research 265 rely on thresholds to describe participants' responses to painful and non-painful stimuli. In 266 contrast, many fields have adopted psychophysical methods, specifically SDT, to describe the

267 performance of sensory systems and, more generally, the performance of diagnostic systems 268 (Swets, 1988).

269 The analysis of MPS data in terms of SDT may also link pain research to contemporary 270 psychological theories of chronic pain and neuroscience. For example the concept of

271 interoception (Craig, 2002), i.e. the ability to correctly perceive internal states, has been 
272 investigated using heartbeat-detection tasks that are based in SDT (Barrett et al., 2004). It may be

273 interesting to contrast these non-nociceptive forms of interceptive awareness to data from

274 nociceptive stimulation. SDT is widely used in cognitive neuroscience to describe non-painful

275 sensory processes. For example, a recent study attempted to link the SDT-parameters to

276 activations in specific brain areas (Reckless et al.). Specifically, these authors found that shifts in

277 response bias were associated with activation in the left dorsolateral prefrontal cortex, which also

278 plays a key role in the modulation of pain (Lorenz, Minoshima \& Casey, 2003). Combining the

279 methods afforded by modern neuroscience and data analysis may yield important new insights

280 into the nature of acute and chronic pain.

281 3.1. Limitations

282 Also two important limitations should be kept in mind. First, applying SDT to pain-

283 ratings requires that the data are collected using the method of constant stimuli, i.e. many

284 different responses to various stimuli in a pseudorandom order need to be recorded. In addition

285 to the disadvantages that are described above, this form of stimulation is only feasible with

286 stimuli that can be presented at different intensities. For modalities such as heat it is much harder

287 to present various intensities in random order. In order to present heat in random order, one

288 would have to lift the thermode after each stimulation, wait until the temperature changes and

289 then re-attach the thermode. Second, a major problem concerns the interpretation of the resulting

290 model effects. As described in the introduction the early differentiation between factor that

291 influence only psychological vs. neurological factors is not adequate (Rollman, 1976; Coppola \&

292 Gracely, 1983; Gracely, 2006). At present we believe it is best to use these parameters

293 descriptively without assuming that the observed differences are due to either "psychologic" or

294 "neurologic" mechanisms. Further research is needed to elucidate the underlying mechanisms 
295 that give rise to the observed differences and it is very likely that several reasons exist that result

296 in a lower sensitivity or response bias. Given the wide use of the QST-protocol we are optimistic

297 that such knowledge will be accumulated over a short period of time.

298

299 4. Conclusions

300 As in all domains of research, progress in pain research is often associated with new

301 methods for data collection or analysis. Although we presented an alternative to thresholds, we

302 would like to stress that we believe that measuring thresholds using the methods of limits is

303 valuable for clinical practice, especially when the effects under study are large and faking is not

304 expected (Gracely, 2006). The approach that we advocate in this paper is a significant extension

305 from earlier work suggesting the use of SDT in pain-research. First, we do not believe that it is

306 possible to uphold the dogma that the response criterion is only influenced by psychological

307 factors while the discrimination parameter is only influenced by physiological factors (Coppola

$308 \&$ Gracely, 1983). Second, we believe that the use of standardized stimulation protocols is a

309 necessary precondition to develop a robust research base into the factors that affect the SDT

310 parameters. Third, we highlight the utility of multilevel models to estimate continuous data

311 simultaneously on the individual and group-level. We hope that the methods presented in the

312 present paper motivate others to utilize these methods in their research.

\section{5. Acknowledgements}

314 Authors declare no conflict of interest. 
316

317

318

319

320

321

322

323

324

325

326

327

328

329

330

331

332

333

334

335

336

337

338

\section{References}

Baayen RH., Davidson DJ., Bates DM. 2008. Mixed-effects modeling with crossed random effects for subjects and items. Journal of memory and language 59:390-412.

Barr DJ., Levy R., Scheepers C., Tily HJ. 2013. Random effects structure for confirmatory hypothesis testing: Keep it maximal. Journal of Memory and Language 68:255-278.

Barrett LF., Quigley KS., Bliss-Moreau E., Aronson KR. 2004. Interoceptive sensitivity and selfreports of emotional experience. Journal of personality and social psychology 87:684.

Bates D., Sarkar D. 2007. lme4: Linear mixed-effects models using S4 classes.

Blankenburg M., Boekens H., Hechler T., Maier C., Krumova E., Scherens A., Magerl W., Aksu F., Zernikow B. 2010. Reference values for quantitative sensory testing in children and adolescents: developmental and gender differences of somatosensory perception. Pain 149:76-88.

Blankenburg M., Meyer D., Hirschfeld G., Kraemer N., Hechler T., Aksu F., Krumova EK., Magerl W., Maier C., Zernikow B. 2011. Developmental and sex differences in somatosensory perception--a systematic comparison of 7-versus 14-year-olds using quantitative sensory testing. Pain 152:2625-2631.

Chapman CR. 1977. Sensory decision theory methods in pain research: a reply to Rollman. Pain $3: 295-305$.

Clark WC. 1974. Pain sensitivity and the report of pain: an introduction to sensory decision theory. Anesthesiology 40:272-287.

Clark WC., Yang JC. 1974. Acupunctural analgesia? Evaluation by signal detection theory. Science 184:1096-1098.

Coppola R., Gracely RH. 1983. Where is the noise in sdt pain assessment? Pain 17:257-266. 
339 Craig AD. 2002. How do you feel? Interoception: the sense of the physiological condition of the $340 \quad$ body. Nature Reviews Neuroscience 3:655-666.

341 DeCarlo LT. 1998. Signal detection theory and generalized linear models. Psychological Methods 3:186.

343 Fillingim RB., King CD., Ribeiro-Dasilva MC., Rahim-Williams B., Riley III JL. 2009. Sex, gender, and pain: a review of recent clinical and experimental findings. The Journal of

Goodenough B., Thomas W., Champion GD., Perrott D., Taplin JE., von Baeyer CL., Ziegler JB. 1999. Unravelling age effects and sex differences in needle pain: ratings of sensory intensity and unpleasantness of venipuncture pain by children and their parents. Pain 80:179-190.

Gracely RH. 2006. Studies of pain in human subjects. In: Wall \& Melzack's Textbook of pain. London: Churchill Livingstone, 267-289.

Green DM., Swets JA. 1966. Signal detection theory and psychophysics. Wiley New York.

Hirschfeld G., Zernikow B., Kraemer N., Hechler T., Aksu F., Krumova E., Maier C., Magerl W., Blankenburg M. 2012. Development of Somatosensory Perception in Children: A Longitudinal QST-Study. Neuropediatrics 43:10-16.

Lorenz J., Minoshima S., Casey KL. 2003. Keeping pain out of mind: the role of the dorsolateral prefrontal cortex in pain modulation. Brain: a journal of neurology 126:1079-1091.

Macmillan NA., Creelman CD. 2004. Detection theory: A user's guide. Lawrence Erlbaum. Magerl W., Krumova EK., Baron R., Tölle T., Treede RD., Maier C. 2010. Reference data for quantitative sensory testing (QST): refined stratification for age and a novel method for statistical comparison of group data. Pain 151:598-605. 
362 Maier C., Baron R., Tölle TR., Binder A., Birbaumer N., Birklein F., Gierthmühlen J., Flor H.,

363

364

365

366

367

368

369

370

371

372

373

374

375

376

377

378

379

380

381

382

383

384 Geber C., Huge V., Krumova EK., Landwehrmeyer GB., Magerl W., Maihöfner C., Richter H., Rolke R., Scherens A., Schwarz A., Sommer C., Tronnier V., Uçeyler N., Valet M., Wasner G., Treede R-D. 2010. Quantitative sensory testing in the German Research Network on Neuropathic Pain (DFNS): somatosensory abnormalities in 1236 patients with different neuropathic pain syndromes. Pain 150:439-450.

Mills C., LeBlond D., Joshi S., Zhu C., Hsieh G., Jacobson P., Meyer M., Decker M. 2012. Estimating Efficacy and Drug ED50's Using von Frey Thresholds: Impact of Weber's Law and Log Transformation. The Journal of Pain 13:519-523.

Mücke M., Cuhls H., Radbruch L., Baron R., Maier C., Tölle T., Treede R-D., Rolke R. 2014. Quantitative sensorische Testung. Der Schmerz 28:635-648.

R Core Team. 2012. R: A Language and Environment for Statistical Computing. $R$ Foundation for Statistical Computing Vienna Austria.

Reckless GE., Bolstad I., Nakstad PH., Andreassen OA., Jensen J. Motivation alters response bias and neural activation patterns in a perceptual decision-making task. Neuroscience.

Rolke R., Baron R., Maier C., Tölle TR., Treede R-D., Beyer A., Binder A., Birbaumer N., Birklein F., Bötefür IC., Braune S., Flor H., Huge V., Klug R., Landwehrmeyer GB., Magerl W., Maihöfner C., Rolko C., Schaub C., Scherens A., Sprenger T., Valet M., Wasserka B. 2006. Quantitative sensory testing in the German Research Network on Neuropathic Pain (DFNS): standardized protocol and reference values. Pain 123:231243.

Rollman GB. 1976. Signal detection theory assessment of pain modulation: a critique. Advances in pain research and therapy 1:355-362. 
385 Rollman GB. 1979. Signal detection theory pain measures: empirical validation studies and $386 \quad$ adaptation-level effects. Pain 6:9-21.

387 Schestatsky P., Stefani LC., Sanches PR., Silva Júnior DP., Torres ILS., Dall-Agnol L., Balbinot 388 LF., Caumo W. 2011. Validation of a Brazilian quantitative sensory testing (QST) device 389 for the diagnosis of small fiber neuropathies. Arquivos de neuro-psiquiatria 69:943-948.

390 Stanislaw H., Todorov N. 1999. Calculation of signal detection theory measures. Behavior 391 Research Methods, Instruments, \& Computers: A Journal of the Psychonomic Society, $392 \quad$ Inc 31:137-149.

393 Swets JA. 1988. Measuring the accuracy of diagnostic systems. Science 240:1285-1293.

394 Wright DB., Horry R., Skagerberg EM. 2009. Functions for traditional and multilevel 395 approaches to signal detection theory. Behavior research methods 41:257-267.

396 Wright DB., London K. 2009. Multilevel modelling: Beyond the basic applications. British Journal of Mathematical and Statistical Psychology 62:439-456. indices and pain threshold in chronic pain patients and healthy volunteers. Psychosomatic medicine 47:461. 
1

Problems associated with the method of limits

(A) Analogue to the method of constant stimuli: Randomized order of stimuli, as in the method of constant stimuli, prohibits any predictions. (B) Analogue to the method of limits: Ordered presentation makes predicting the next stimulus extremely easy.

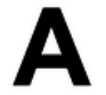

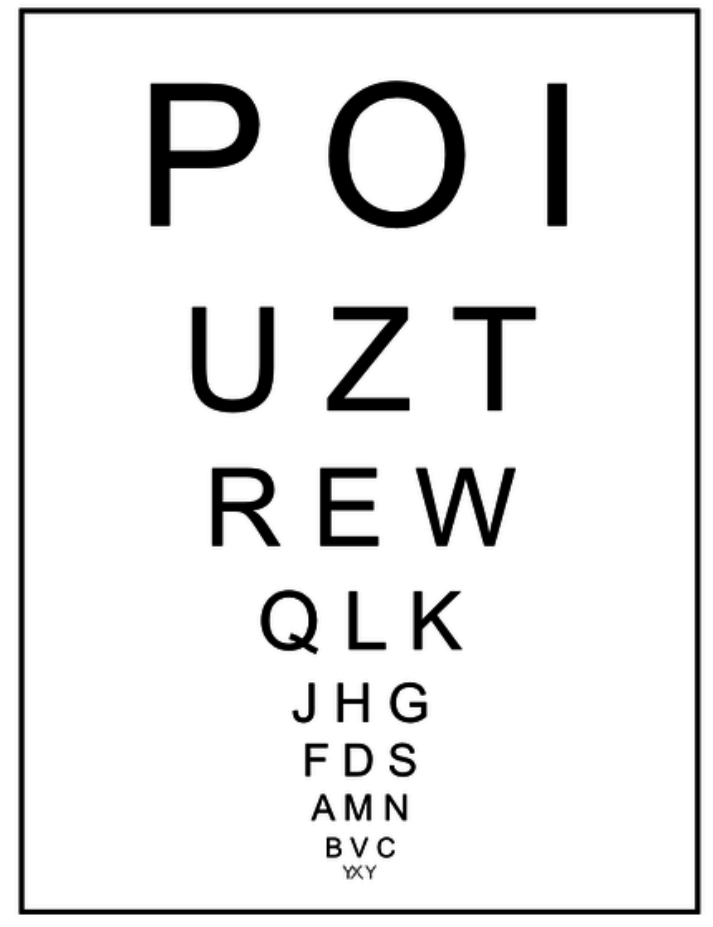

Read aloud from top to bottom.

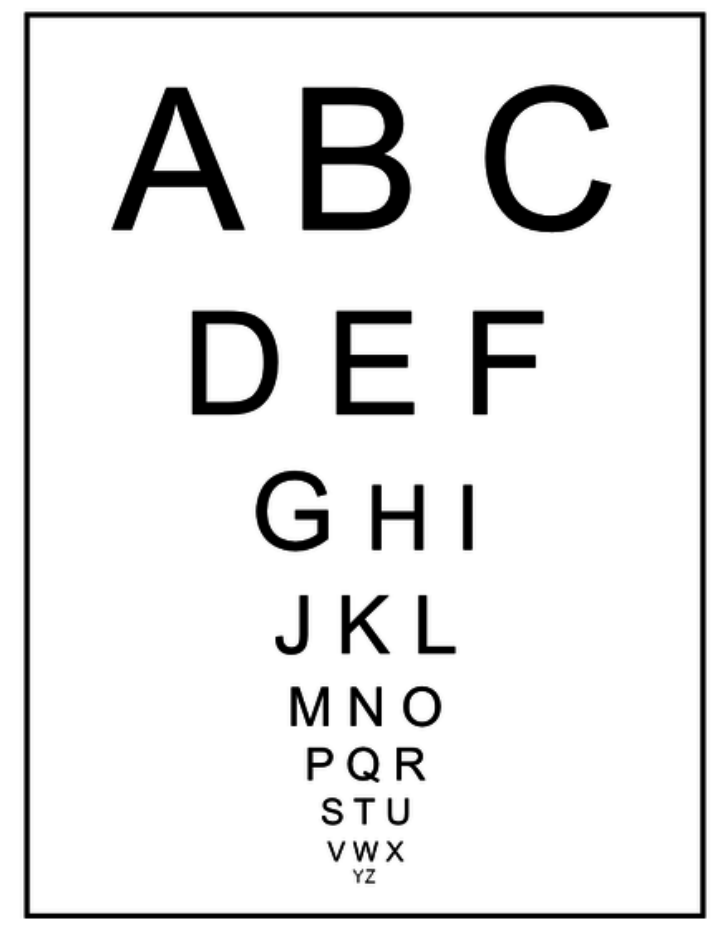

Read aloud from top to bottom. 
2

Responses and fitted model for one subject

Participants rated stimuli at different intensities. Note: Points represent the average $\%$ of responses painful at each stimulus intensity, the blue line indicates the fit to the individual data 


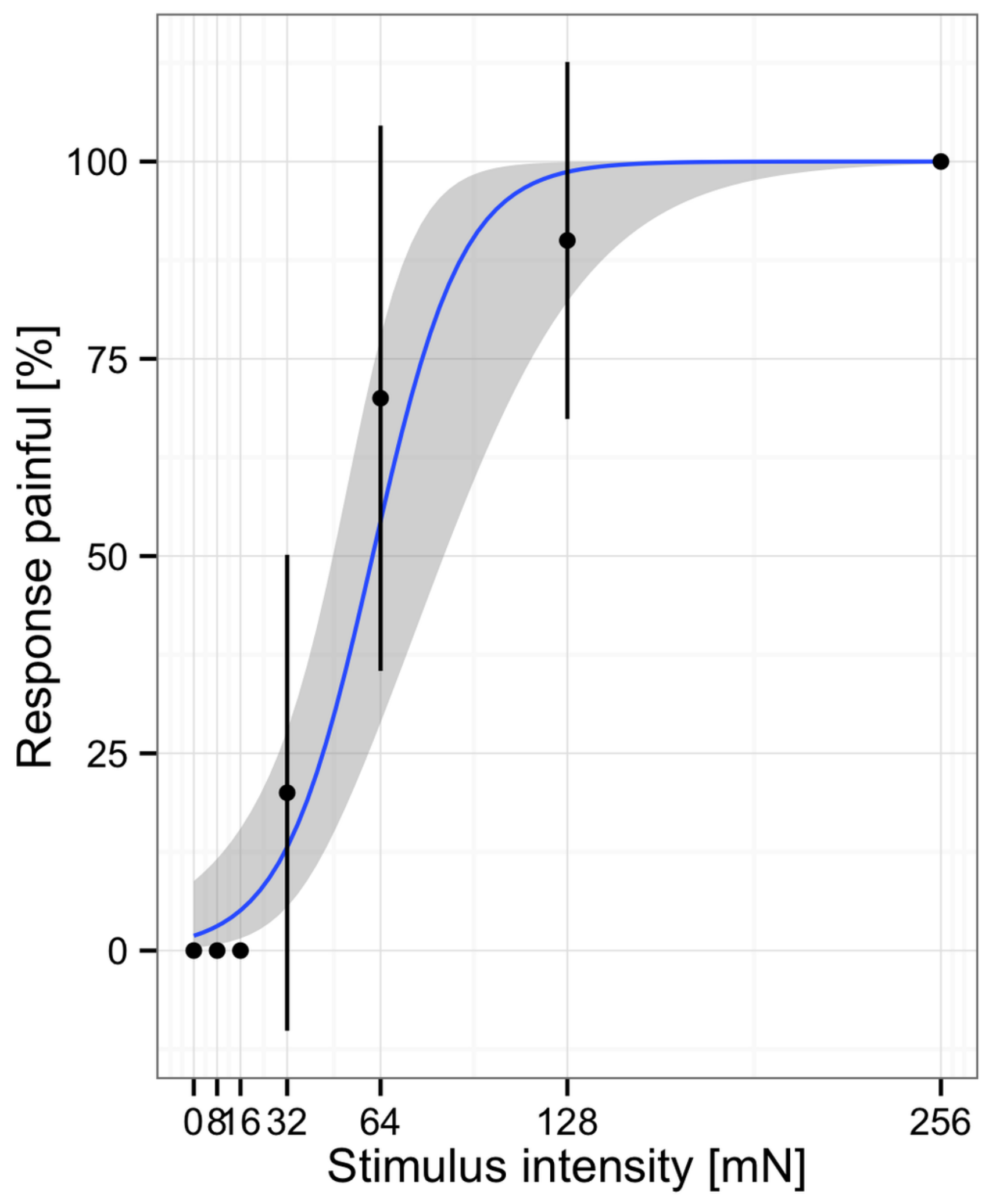


3

Responses of individual participants

Each panel represents an individual, points represent the average \% of responses painful at each stimulus intensity, the blue line indicates the fit to the individual data. 

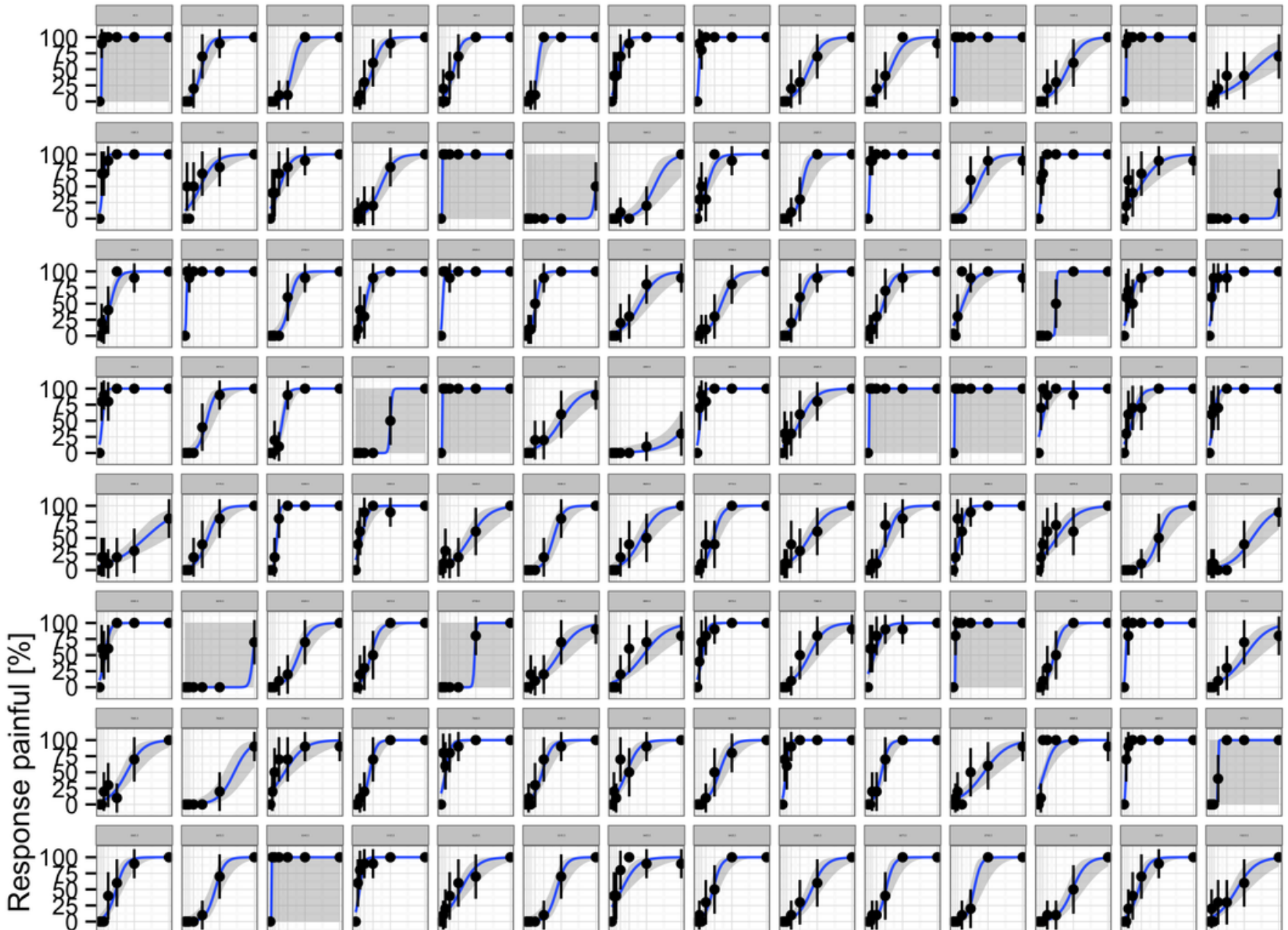

$\begin{array}{ll}20 & =1 \\ 25 & =\$ 2\end{array}$
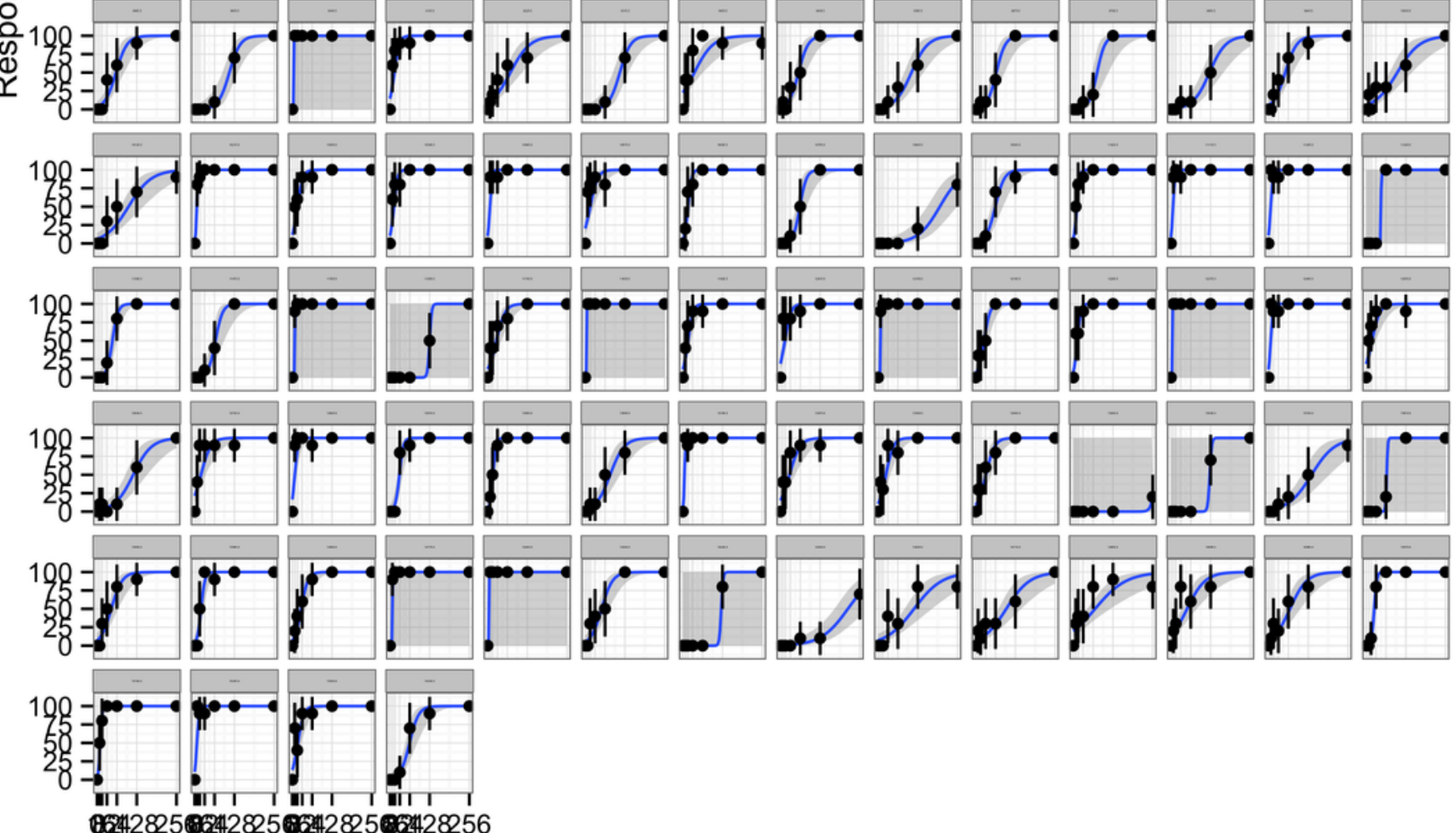

- 242825

Stimulus intensity [mN] 


\section{Table $\mathbf{1}$ (on next page)}

Important functions to fit GLMs to psychophysical data 
1 Table 1. Important functions to fit GLMs to psychophysical data.

\begin{tabular}{ll}
\hline \multicolumn{1}{c}{ Function } & \multicolumn{1}{c}{ What it does } \\
\hline glmer() & Fits a model to data. The example code specifies a dependent \\
& variable "res_01", fixed effects "Stimulus", random effects \\
& "(Stimulus|Code)", a link function "binomial(link=logit)", and the \\
& data to which this is fitted. \\
& Example: \\
& $>$ mod_01<-glmer(res_01 $\sim$ Stimulus + (Stimulus|Code), family $=$ \\
& binomial(link=logit), data $=$ data) \\
& Compares fitted models to each other. The example code compares \\
& three consecutively more complex models. \\
& Example: \\
& $>$ anova(mod_01, mod_02, mod_03) \\
& Gives an overview of the model, including parameter estimates and \\
& significance levels. \\
Example:
\end{tabular}

2 
Table 2 (on next page)

Series of model comparisons 
1 Table 2. Series of model comparisons

\begin{tabular}{llll}
\hline Model & $\chi^{2}\left(\operatorname{diff} \chi^{2}\right)$ & DF (diff DF) & $p\left(\chi^{2}\right)$ \\
\hline M1: Baseline & & & \\
M2: Model M1 plus stimulus & $(94.7011)$ & 1 & $<.001$ \\
M3: Model M2 plus sex and stimulus * sex & $(1.7872)$ & 2 & 0.41 \\
M4: Model M2 plus age and stimulus * age & $(8.847)$ & 2 & .01 \\
\hline
\end{tabular}

2 


\section{Table $\mathbf{3}$ (on next page)}

Estimates of the final model (M4) 
1 Table 3. Estimates of the final model (M4)

\begin{tabular}{llcccc}
\hline Parameter & Estimate & SE & OR & $z$ & $p$ \\
\hline Intercept & -3.29 & .14 & 0.04 & -23.11 & $<.001$ \\
Stimulus & 0.10 & .02 & 1.11 & 6.20 & $<.001$ \\
Age (Children) & 0.14 & .20 & 1.15 & .72 & .473 \\
Stimulus * Age & .07 & .02 & 1.07 & 2.97 & .003 \\
\hline
\end{tabular}

2 Note: Reference category in parenthesis 